\title{
Ganglioside GT1b increases hyaluronic acid synthase 2 via PI3K activation with TLR2 dependence in orbital fibroblasts from thyroid eye disease patients
}

\author{
Hyun Kyu Yoo ${ }^{1, \#}$, Hyunju Park ${ }^{2, \#}$, Hye Suk Hwang ${ }^{1}$, Hee Ja Kim ${ }^{2}$, Youn-Hee Choi ${ }^{2}$ \& Koung Hoon Kook ${ }^{1, *}$ \\ ${ }^{1}$ Department of Ophthalmology, Ajou University School of Medicine, Suwon 16499, ${ }^{2}$ Department of Physiology, Inflammation-Cancer \\ Microenvironment Research Center, Ewha Womans University School of Medicine, Seoul 07804, Korea
}

\begin{abstract}
Thyroid eye disease (TED) is a complex autoimmune disease with a spectrum of signs. we previously reported that trisialoganglioside (GT)1b is significantly overexpressed in the orbital tissue of TED patients, and that exogenous GT1b strongly induced HA synthesis in orbital fibroblasts. However, the signaling pathway in GT1b-induced hyaluronic acid synthase (HAS) expression in orbital fibroblasts from TED patients have rarely been investigated. Here, we demonstrated that GT1b induced phosphorylation of Akt/mTOR in a dose-dependent manner in orbital fibroblasts from TED patients. Both co-treatment with a specific inhibitor for PI3K and siRNA knockdown of TLR2 attenuated GT1b-induced Akt phosphorylation. GT1b significantly induced HAS2 expression at both the transcriptional and translational level, which was suppressed by specific inhibitors of PI3K or Akt/mTOR, and by siRNA knockdown of TLR2. In conclusion, GT1b induced HAS2 in orbital fibroblasts from TED patients via activation of the PI3Krelated signaling pathway, dependent on TLR2. [BMB Reports 2021; 54(2): 136-141]
\end{abstract}

\section{INTRODUCTION}

Thyroid eye disease (TED) is an autoimmune disorder in which stimulatory antibodies bind to the thyrotropin receptor, resulting in active inflammation of orbital tissues such as orbital fat and extraocular muscle (1). While most TED patients experience only mild ocular surface discomfort, approximately $3-5 \%$ of patients suffer from more severe ophthalmopathy, characterized by proptosis, eyelid retraction, chemosis, double vision, limitation of ocular

*Corresponding author. Tel: +82-31-219-5260; Fax: +82-31-219-5259; E-mail: drkook@ajou.ac.kr

${ }^{\#}$ These authors contributed equally to this work.

https://doi.org/10.5483/BMBRep.2021.54.2.178

Received 26 August 2020, Revised 10 September 2020, Accepted 17 December 2020

Keywords: Orbital fibroblast, Phosphoinositide 3-kinase, Thyroid eye disease, Toll-like receptor 2, Trisialoganglioside $1 \mathrm{~b}$ motion, and even vision loss due to corneal ulceration or compressive optic neuropathy $(2,3)$. Most of these clinical findings result from progressive enlargement of orbital tissues within the limited space of the bony orbit (4). Although the pathogenesis of TED is not completely understood, the volumetric expansion of orbital tissues is known to originate from edematous changes of connective tissue and extraocular muscles, which is caused by extracellular accumulation of hyaluronic acid (HA) (5).

$\mathrm{HA}$, which belongs to the glycosaminoglycan family, is one of the most prominent macromolecules of extracellular matrices. It is synthesized by a class of integral membrane proteins called hyaluronic acid synthase (HAS), of which vertebrates have three types: HAS1, HAS2, and HAS3. These enzymes lengthen hyaluronan by repeatedly adding glucuronic acid and $\mathrm{N}$-acetylglucosamine to the nascent polysaccharide as it is extruded via an ABC-transporter through the cell membrane into the extracellular space (6), which occurs according to the rheological properties of HA including hydrophilia, polyanionic charge, and high osmotic pressure.

The extracellular accumulation of HA attracts water into the tissue, resulting in edema (7). Taken together with the characteristics of orbital fibroblasts and resident cells in orbital fatty connective tissue, which possess the capability to produce a significant amount of $\mathrm{HA}$ in response to proinflammatory cytokines such as interleukin (IL)- $1 \beta$ and transforming growth factor (TGF)- $\beta$ (8-10), the effective suppression of HA production in orbital fibroblasts is thought to be a therapeutic target in TED.

Ganglioside is an acidic glycosphingolipid that has a variable sialic acid-containing oligosaccharide structure attached to an acylated ceramide core (11). More than 60 gangliosides exist, according to the numbers and sites of sialic acid residues. They are classified into a-, b-, and c- series gangliosides according to the numbers and sites of sialic acids bound to the galactose portion of the molecule (12). The a-series includes monosialoganglioside (GM)3, GM2, GM1, disialoganglioside (GD)1a, and trisialoganglioside (GT)1a; the b-series includes GD3, GD2, GD1b, GT1b, and quatrosialoganglioside (GQ) 1b; and the c-series includes GT3, GT2, GT1c, GQ1c, and pentasialoganglioside (GP)1c (13). They are widely distributed in the plasma membrane

ISSN: 1976-670X (electronic edition)

Copyright (C) 2021 by the The Korean Society for Biochemistry and Molecular Biology

(c) This is an open-access article distributed under the terms of the Creative Commons Attribution Non-Commercial License (http://creativecommons.org/licenses/by-nc/4.0) which permits unrestricted non-commercial use, distribution, and reproduction in any medium, provided the original work is properly cited. 
of vertebrate tissue and are known to play a key role in cell differentiation, adhesion, proliferation, and signal transduction (14). In our previous study, we reported that the expression of GT1b was increased in the orbital fatty connective tissues of TED patients, and that GT1b induced an increase of both HAS and $\mathrm{HA}$ in orbital fibroblasts, suggesting a possible role of gangliosides in the pathogenesis of TED (15). However, the signaling pathway of GT1b, which could be a potential therapeutic target, has not been completely elucidated.

In the present study, we describe a signaling pathway through which GT1b increased HAS2 expression in orbital fibroblasts from TED patients. We showed that GT1b induced phosphorylation of both protein kinase B (Akt) and mammalian target of rapamycin (mTOR) via phosphoinositide 3-kinase (PI3K) activation; moreover, the increased HAS2 expression induced by GT1b was effectively attenuated by co-treatment with specific inhibitors of PI3K or mTOR in orbital fibroblasts from TED patients. Additionally, we showed that loss of Toll-like receptor (TLR)2 attenuated the GT1b-induced HAS2 increase in cells. Taken together, our results indicated that GT1b induced HAS2 expression by activating the PI3K/Akt/mTOR signaling cascade, with dependence on the TLR2. Our results provide insight into the signaling pathways through which GT1b stimulates HA synthesis in orbital fibroblasts from TED patients, suggesting a possible specific therapeutic target for the treatment of this disease.

\section{RESULTS}

\section{PI3K inhibitor reduced GT1b-induced Akt/mTOR phosphorylation}

We initially examined whether the Akt/mTOR signaling pathway was activated in response to GT1b in orbital fibroblasts from TED patients. Cells were treated by commercially available GT1b with graded concentrations up to $40 \mu \mathrm{g} / \mathrm{ml}$ for 5 minutes. GT1b induced a dose-dependent increase in the levels of phosphorylated Akt up to a concentration of $40 \mu \mathrm{g} / \mathrm{ml}$, with no changes in the amount of Akt or GAPDH proteins (Fig. 1A). A significant increase in Akt phosphorylation was detected at 5 minutes in orbital fibroblasts from TED patients treated with 40 $\mu \mathrm{g} / \mathrm{ml} \mathrm{GT1b}$, which showed a gradual decrease with time but persisted at a significant level for up to 30 minutes (Fig. 1B). The effects of GT1b on mTOR phosphorylation were also examined. Immunoblot analyses revealed that mTOR phosphorylation reached significantly increased levels at 30 minutes after GT1b treatment (Fig. 1C). The Akt/mTOR signaling pathways are known to be activated mainly by PI3K (16). To examine whether GT1b-induced phosphorylation of Akt/mTOR was mediated by PI3K in orbital fibroblasts from TED patients, we utilized a PI3K inhibitor (LY294002). GT1b-induced phosphorylation of Akt was significantly blocked in orbital fibroblasts from TED patients by LY294002 in a dose-dependent manner, up to a concentration of $10 \mu \mathrm{g} / \mathrm{ml}$ (Fig. 1D). Both Akt and mTOR were phosphorylated in response to GT1b $(40 \mu \mathrm{g} / \mathrm{ml})$; this GT1b-induced phosphorylation of Akt/mTOR was significantly reduced
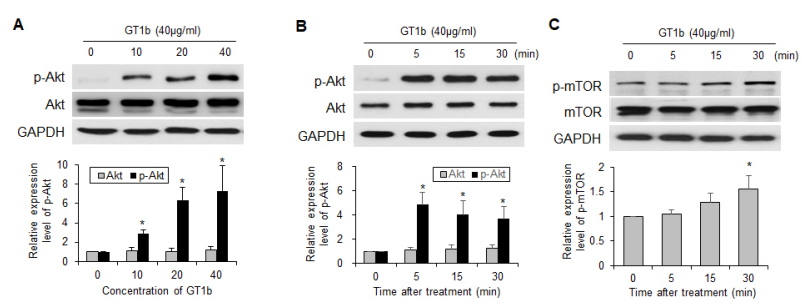

D
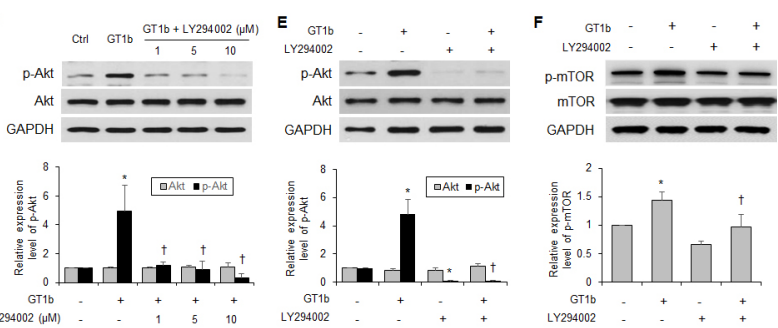

Fig. 1. GT1b-induced Akt/mTOR phosphorylation was mediated by phosphoinositide 3-kinase in orbital fibroblasts from thyroid eye disease (TED) patients. Orbital fibroblasts were cultivated from TED patients $(n=5)(A)$ Cells were treated with gradual increments of GT1b $(10,20,40 \mu \mathrm{g} / \mathrm{ml})$ for 5 minutes, followed by immunoblotting for phosphorylated Akt (p-Akt) or Akt. (B, C) Following treatment of cells with $40 \mu \mathrm{g} / \mathrm{ml}$ GT1b for the indicated times, immunoblotting was performed for $\mathrm{p}$-Akt/Akt and p-mTOR/mTOR, respectively. (D) Cells were treated with $40 \mu \mathrm{g} / \mathrm{ml}$ of GT1b with or without addition of gradual increments of LY294002 (1, 5, and $10 \mu \mathrm{M})$ for 5 minutes, followed by immunoblotting for p-Akt or Akt. (E) Cells were treated with $40 \mu \mathrm{g} / \mathrm{ml}$ of GT1b in the presence or absence of $10 \mu \mathrm{M}$ of LY294002 for 5 minutes and immunoblotting was performed for p-Akt or Akt. (F) Following treatment with GT1b and/or LY294003 for 30 minutes as in (E), immunoblotting was performed for phosphorylated mTOR. Representative blots from independent experiments, for cells from each TED patient, are shown. Quantification of each protein (fold increase) was calculated by dividing the densitometric value of each lane by the corresponding glyceraldehyde 3-phosphate dehydrogenase $(\mathrm{GAPDH})$ value (bar graphs, means $\pm \mathrm{SD}$; $* \mathrm{P}<0.05$ vs. untreated control; ${ }^{\top} \mathrm{P}<0.05$ vs. GT1b-treated cells).

by LY294002 (Fig. 1E, F), indicating that PI3K was directly involved in the GT1b-induced phosphorylation of Akt/mTOR signaling pathways in orbital fibroblasts from TED patients.

\section{Akt phosphorylation by GT1b was mediated by TLR2}

Because there have been reports suggesting that TLR2 is involved in gangliosides-mediated intracellular signaling (17), and that TLR2 stimulation activates PI3K/Akt signaling (18), we next examined whether downregulation of TLR2 expression using specific siRNA affected Akt phosphorylation in orbital fibroblasts from TED patients. Fig. 2A, B shows that transfection of cells with TLR2 siRNA decreased endogenous TLR2 expression. In the presence of TLR2 siRNA, GT1b failed to induce an increase in Akt phosphorylation (Fig. 2C), which supported the hypothesis that GT1b induced Akt phosphorylation and activation via TLR2. 
A
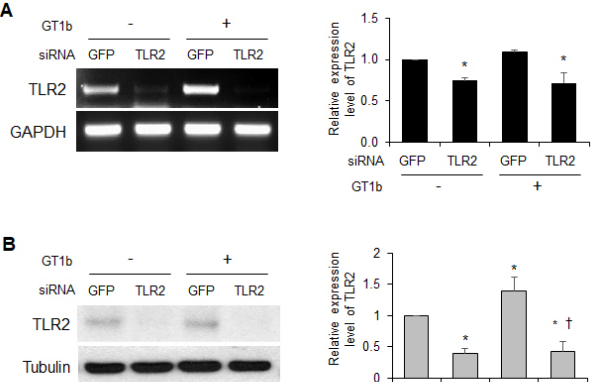

C
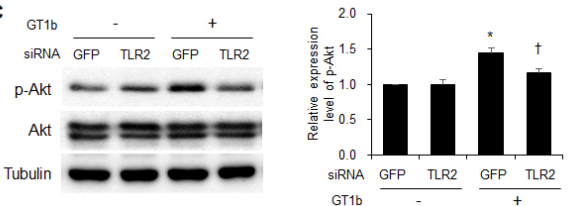

Fig. 2. Akt phosphorylation by GT1b was mediated by Toll-like receptor (TLR)2. (A, B) Orbital fibroblasts were transiently transfected with TLR2 siRNA or control siRNA. One day after transfection, cells were treated with or without GT1b $(40 \mu \mathrm{g} / \mathrm{ml})$ for 24 hours and analyzed by reverse transcription-polymerase chain reaction (RT-PCR) and immunoblotting. (C) Cells transiently transfected with TLR2 siRNA or control siRNA were treated with GT1b $(40 \mu \mathrm{g} / \mathrm{ml})$ for $0,0.5$, $1.5,3$, and 6 hours and analyzed by immunoblotting with phosphorylation-specific antibodies for Akt. Effective siRNA-mediated suppression of TLR2 mRNA/protein expression was verified for each assay by RT-PCR and immunoblotting. Tubulin was used as the loading control. Data are presented as the fold increase compared with untreated control cells. Data shown are representative of at least three experiments (bar graphs, means $\pm \mathrm{SD} ; * \mathrm{P}<0.05$ vs. untreated control; ${ }^{\dagger} \mathrm{P}<0.05$ vs. GT1b-treated cells).

\section{The PI3K/mTOR inhibitors abrogated GT1b-induced HAS expression}

We previously reported that GT1b induced HA production in orbital fibroblasts, which might have resulted from increased expression of HAS by GT1b (15). We therefore examined the contribution of PI3K to GT1b-induced HAS expression by utilizing a PI3K inhibitor. To determine whether LY294002 affected HAS expression at the transcriptional level, orbital fibroblasts from TED patients were treated with GT1b $(40 \mu \mathrm{g} / \mathrm{ml})$ in the presence or absence of LY294002 $(10 \mu \mathrm{g} / \mathrm{ml})$ for 24 hours or 48 hours, and RNA was then extracted and subjected to qRTPCR. At 24 hours of incubation, the expression levels of HAS1, HAS2, and HAS3 were increased by GT1b treatment, and attenuated by co-treatment with LY294002, but the changes were not significant (Fig. 3A, left). At 48 hours of incubation, the expression level of HAS2 was significantly increased by GT1b treatment, and inhibition of PI3K suppressed GT1b-induced HAS2 expression. HAS1 and HAS3 showed similar results as those at 24 hours (Fig. 3A, right). These results were verified by RT-PCR, which showed that GT1b increased mRNA transcription of HAS1, HAS2 and HAS3, and LY294002 dramatically

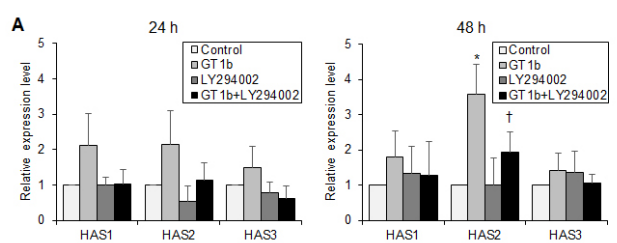

B
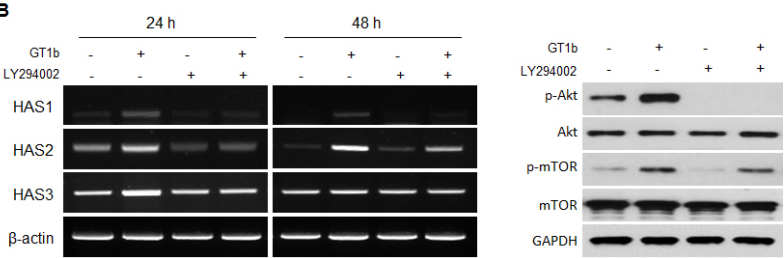

C
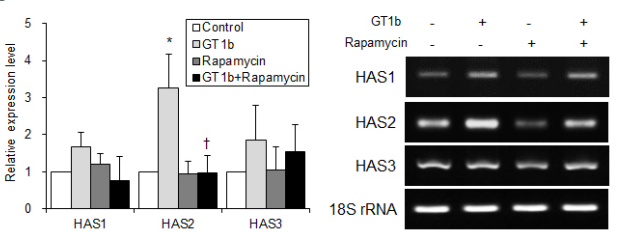

Fig. 3. Involvement of the PI3K/Akt/mTOR signaling pathway in GT1binduced hyaluronic acid synthase (HAS) transduction. (A) Orbital fibroblasts from TED patients were treated with $40 \mu \mathrm{g} / \mathrm{ml}$ of GT1b/ $10 \mu \mathrm{M}$ or LY294002, alone or in combination at the same dose, for 24 hours or 48 hours and transcriptional levels of HAS1, HAS2, and HAS3 in cells were measured by the quantitative real-time polymerase chain reaction ( $\mathrm{qRT}-\mathrm{PCR}$ ). (B) Following the same treatment protocol as (A), RT-PCR analyses for HAS1, HAS2, or HAS3 were performed. Cells were treated with $40 \mu \mathrm{g} / \mathrm{ml}$ of GT1b in the presence or absence of $10 \mu \mathrm{M}$ of LY294002 and immunoblotting was performed for $\mathrm{p}$-Akt/Akt and p-mTOR/mTOR. (C) Cells were treated with $40 \mu \mathrm{g} / \mathrm{ml}$ of $\mathrm{GT} 1 \mathrm{~b} / 5 \mathrm{ng} / \mathrm{ml}$ of rapamycin, alone or in combination at the same dose, for 48 hours and the transcriptional levels of HAS1, HAS2, and HAS3 in cells were measured by qRT-PCR (left) or RT-PCR (right). Representative blots from independent experiments for cells from each TED patient are shown. ${ }^{*} \mathrm{P}<0.05$ vs. untreated control; ${ }^{+} \mathrm{P}<0.05$ vs. GT1b-treated cells.

suppressed the GT1b-induced increase in HAS mRNA levels; this was the most striking for HAS2 (Fig. 3B, left). LY294002 alone did not show suppression of HAS mRNA levels. GT1b-induced phosphorylation of Akt/mTOR was inhibited by LY294002 (Fig. 3B, right).

To explore whether activation of mTOR was involved in the signaling pathway of GT1b-induced PI3K-mediated HAS expression, qRT-PCR was performed using rapamycin, a specific inhibitor of mTOR. Fig. 3C (left) shows that GT1b strikingly increased HAS2 transcription, and co-treatment with rapamycin drastically suppressed it. Furthermore, these results were verified by RT-PCR (Fig. 3C, right). Taken together, these results indicated that GT1b induced expression of HAS2 through the PI3K/Akt/mTOR signaling pathways in orbital fibroblasts from TED patients. 


\section{Loss of TLR2 and PI3K/mTOR inhibitors attenuated GT1b-induced HAS2 transcription and translation, respectively}

Orbital fibroblasts transfected with TLR2 siRNA displayed reduced levels of endogenous TLR2 expression, as confirmed by RT-PCR (data not shown), in a manner similar to Fig. 2A. In the presence of TLR2 siRNA, GT1b failed to induce an increase in HAS2 mRNA/protein expression (Fig. 4A, B). Because transcription of HAS2 was substantially elevated in GT1b-treated orbital fibroblasts from TED patients, and this increase was significantly suppressed by LY294002 as well as rapamycin, we measured relevant protein levels of HAS2 in cell lysates of GT1b-treated orbital fibroblasts using immunoblotting. Consistent with the qRT-PCR and RT-PCR results, phosphorylation of Akt/mTOR and HAS2 protein expression was enhanced by GT1b treatment and suppressed by LY294002 and rapamycin treatments,

A
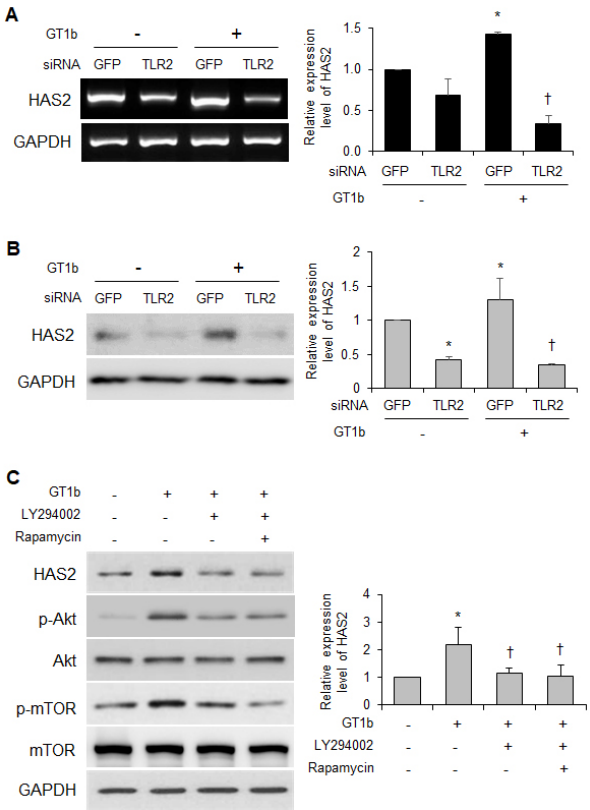

Fig. 4. Dependence on TLR2 and $P I 3 K / A k t / m T O R$ signaling pathway in GT1b-induced HAS2 expression. (A, B) Orbital fibroblasts were transiently transfected with TLR2 siRNA or control siRNA. One day after transfection, cells were treated with or without GT1b $(40 \mu \mathrm{g})$ $\mathrm{ml}$ ) for 24 hours and analyzed by the RT-PCR and immunoblotting. Values for HAS2 mRNA/protein expression were normalized to GAPDH levels for each experimental condition. The value of HAS2 in the untreated control transfected with control siRNA was set as 1. (C) Cells were treated with $40 \mu \mathrm{g} / \mathrm{ml}$ of GT1b with or without addition of $10 \mu \mathrm{M}$ of LY294002 or $5 \mathrm{ng} / \mathrm{ml}$ of rapamycin, and immunoblotting was performed for HAS2, p-Akt/Akt and p-mTOR/mTOR. Representative blots from independent experiments, for cells from each TED patient, are shown. The data represent three independent experiments. Quantification of each protein (fold increase) was calculated by dividing the densitometric value of each lane by the corresponding GAPDH value (bar graphs, means $\pm \mathrm{SD} ; * \mathrm{P}<0.05$ vs. untreated control; ${ }^{\dagger} \mathrm{P}<0.05$ vs. GT1b-treated cells). respectively (Fig. 4C).

\section{DISCUSSION}

Ganglioside is an acidic glycosphingolipid that can be classified according to the number of sialic acid residues. Ganglioside is known to play important roles in the nervous system, including neuronal plasticity and regeneration. Experimental data have shown that exogenous gangliosides exhibit properties similar to neurotrophins, which are important in the survival and maintenance of neurons (19). Among them, increasing evidence has shown that the b-series ganglioside, GT1b, has a potential role in modulating the immunological process. For example, GT1b indirectly suppresses immunoglobulin production in human peripheral blood mononuclear cells without affecting the viability of the cells, indicating that GT1b may act as an important inhibitor of the human humoral immune response (20). In another report, the b-series gangliosides, including GT1b, enhanced IL-2 and IFN- $\gamma$ production in phytohemagglutinin-stimulated human T cells (21). Regarding the pathogenic role of gangliosides in TED, we previously reported that GT1b is significantly overexpressed in the orbital tissue of TED patients, and that exogenous GT1b strongly induced HA synthesis in orbital fibroblasts; this suggests a pathological role of GT1b in the pathogenesis of TED (15). In terms of developing a selective molecular treatment for a particular disease based on its pathogenic mechanism, identifying the related signaling pathway is important.

In the present study, GT1b induced Akt phosphorylation by PI3K activation in orbital fibroblasts from TED patients. The PI3K/Akt signaling pathway is an intracellular signaling pathway that is closely associated with cell proliferation, transformation, metabolism, motility, and the development and progression of tumors (22). Regarding its role in pathogenesis, clinical trials for some cancers have been performed using PI3K inhibitors as therapeutic agents, which have shown promising results (23-25). Akt is also known as an important mediator of insulin-activated insulin-like growth factor (IGF)-1 receptor signaling, which plays a significant role in the regulation of adipocyte differentiation (26). Regarding orbital fibroblasts, several stimulants, such as IGF-1 (27), thyroid stimulating antibody (28), and thyroid stimulating hormone (29), have been reported to induce phosphorylation of Akt, suggesting pathogenic roles of its signaling cascade in TED, as well as its potential as a target for novel TED therapies by suppression of the PI3K/Akt signaling pathway. Our results showing that LY294002, a specific PI3K inhibitor, effectively attenuated GT1b-induced Akt phosphorylation in orbital fibroblasts from TED patients supported this possibility.

TLRs are evolutionarily conserved receptors belonging to a family of pattern recognition receptors (30). They are expressed by a variety of cell types, and their activation triggers an inflammatory response by activating regulation pathways of innate and adaptive immunities. However, aberrant activation of TLR signaling pathways leads to the development of chronic inflammatory diseases (31). Regarding ganglioside-mediated intracellular 
signaling, the involvement of TLR2 has been proposed, where brain ganglioside mixtures were shown to enhance the expression of TLR2 in murine microglia (BV2) (17). The relevance of TLR2 in PI3K/Akt signaling pathways was revealed by another study of the signaling cascade through which Pam3Cys (a TLR2 agonist) induces cellular proliferation, including PI3K/Aktmediated proliferation in human colorectal cancer cells (18). Consistent with these two previous reports, our results showed that GT1b induced activation of the PI3K/Akt signaling cascade, and the induction of HAS expression was dependent on TLR2, suggesting a possible link between gangliosides, TLR2, and PI3K/Akt $(17,18)$. To our knowledge, there has been no previous report of the participation of TLR2 in the pathogenesis of TED.

$\mathrm{HA}$, a high molecular weight glycosaminoglycan, is known as an important mediator of TED. Because HA-accumulated tissues can expand by hydration due to the remarkable hydrophilic nature of $\mathrm{HA}$, abnormal $\mathrm{HA}$ accumulation in orbital tissues is considered the hallmark of the connective tissue remodeling found in TED patients (5). In addition, we suggested an additional role of HA in the pathogenesis of TED in our previous report, suggesting that $\mathrm{HA}$ increased COX-2 expression in orbital fibroblasts from TED patients via CD44 through MAPKand NF-kB-mediated signaling pathways, which in turn suggested that $\mathrm{HA}$ had a proinflammatory role in addition to its known role in the swelling of orbital tissue during the pathogenesis of TED (32). HA production is mediated by HAS, a membrane-bound enzyme. There are three known isoforms, HAS1, 2, and 3, in mammalian cells. Although human orbital fibroblasts express low levels of all three HA synthases, HAS2 is the most abundantly expressed isoform (33). In addition, HAS2 protein is strongly increased by db-cAMP in orbital preadipocytes, while HAS1 is undetectable by the same treatment (34). In accordance with these previous reports, GT1b strikingly induced HAS2 mRNA expression, while it did not show a significant effect on HAS1 or HAS3 mRNA expression in our system. These results suggested that HAS2 had a role in the production of HA in orbital tissue of TED patients.

Involvement of Akt signaling in the HAS2 expression of various cells was suggested by previous studies. In dermal fibroblasts (35), fibroblasts from Smpd3 gene-deleted mouse (36), and breast cancer cell line MCF7 (37), the increased expression of HAS2 was positively regulated by phosphorylation of Akt. Consistent with these previous findings, our results showed that GT1b-induced HAS2 expression was mediated by activation of the PI3K/Akt pathway. This activated pathway mediated downstream responses by phosphorylating a range of intracellular target proteins, including mTOR, which is also a member of the PI3K protein kinase family and an important downstream component of the PI3K pathway (38). Our data showing attenuation of GT1b-induced HAS2 expression by co-treatment with a specific mTOR inhibitor supported this possible involvement of the PI3K/Akt/mTOR signal cascade.

In conclusion, our results provided evidence that GT1b activated the PI3K/Akt/mTOR signaling pathways, with depen- dence on TLR2 when inducing HAS2, further suggesting that it could be a pathogenesis-based therapeutic target for TED.

\section{MATERIALS AND METHODS}

Detailed information is provided in Supplementary Information.

\section{ACKNOWLEDGEMENTS}

This study was supported by Basic Science Research Program through the National Research Foundation of Korea (NRF) funded by the Ministry of Science, ICT \& Future Planning (NRF-2016R 1A2B1014704) (K.H.K) and by RP-Grant 2018 of Ewha Womans University (H.P.).

\section{CONFLICTS OF INTEREST}

The authors have no conflicting interests.

\section{REFERENCES}

1. Kazim M, Goldberg RA and Smith TJ (2002) Insights into the pathogenesis of thyroid-associated orbitopathy: evolving rationale for therapy. Arch Ophthalmol 120, 380-386

2. Garrity JA and Bahn RS (2006) Pathogenesis of graves ophthalmopathy: implications for prediction, prevention, and treatment. Am J Ophthalmol 142, 147-153

3. Naik VM, Naik MN, Goldberg RA, Smith TJ and Douglas RS (2010) Immunopathogenesis of thyroid eye disease: emerging paradigms. Surv Ophthalmol 55, 215-226

4. Bahn RS (2010) Graves' ophthalmopathy. N Engl J Med 362, 726-738

5. Smith TJ, Bahn RS and Gorman CA (1989) Connective tissue, glycosaminoglycans, and diseases of the thyroid. Endocr Rev 10, 366-391

6. Schulz T, Schumacher U and Prehm P (2007) Hyaluronan export by the $A B C$ transporter MRP5 and its modulation by intracellular cGMP. J Biol Chem 282, 20999-21004

7. Hansen C, Rouhi R, Forster G and Kahaly GJ (1999) Increased sulfatation of orbital glycosaminoglycans in Graves' ophthalmopathy. J Clin Endocrinol Metab 84, 14091413

8. Korducki JM, Loftus SJ and Bahn RS (1992) Stimulation of glycosaminoglycan production in cultured human retroocular fibroblasts. Invest Ophthalmol Vis Sci 33, 2037-2042

9. Wong YK, Tang KT, Wu JC, Hwang JJ and Wang HS (2001) Stimulation of hyaluronan synthesis by interleukin-1beta involves activation of protein kinase $C$ betall in fibroblasts from patients with Graves' ophthalmopathy. J Cell Biochem $82,58-67$

10. Wang HS, Tung WH, Tang KT et al (2005) TGF-beta induced hyaluronan synthesis in orbital fibroblasts involves protein kinase $C$ betall activation in vitro. J Cell Biochem 95, 256267

11. Svennerholm L (1964) The Gangliosides. J Lipid Res 5, 145155

12. Nakamura K, Inagaki $F$ and Tamai $Y$ (1988) A novel gang- 
lioside in dogfish brain. Occurrence of a trisialoganglioside with a sialic acid linked to $\mathrm{N}$-acetylgalactosamine. J Biol Chem 263, 9896-9900

13. Yu RK, Tsai YT, Ariga T and Yanagisawa M (2011) Structures, biosynthesis, and functions of gangliosides-an overview. J Oleo Sci 60, 537-544

14. Huwiler A, Kolter T, Pfeilschifter J and Sandhoff K (2000) Physiology and pathophysiology of sphingolipid metabolism and signaling. Biochim Biophys Acta 1485, 63-99

15. Kook KH, Choi YH, Kim YR et al (2011) Altered ganglioside expression modulates the pathogenic mechanism of thyroid-associated ophthalmopathy by increase in hyaluronic acid. Invest Ophthalmol Vis Sci 52, 264-273

16. Cantley LC (2002) The phosphoinositide 3-kinase pathway. Science 296, 1655-1657

17. Yoon HJ, Jeon SB, Suk K, Choi DK, Hong YJ and Park EJ (2008) Contribution of TLR2 to the initiation of ganglioside-triggered inflammatory signaling. Mol Cells 25, 99-104

18. Liu YD, Ji CB, Li SB et al (2018) Toll-like receptor 2 stimulation promotes colorectal cancer cell growth via PI3K/Akt and NF-kappaB signaling pathways. Int Immunopharmacol 59, 375-383

19. Lim ST, Esfahani K, Avdoshina V and Mocchetti I (2011) Exogenous gangliosides increase the release of brain-derived neurotrophic factor. Neuropharmacology 60, 1160-1167

20. Kanda N and Tamaki K (1999) Ganglioside GT1b suppresses immunoglobulin production by human peripheral blood mononuclear cells. Immunology 96, 628-633

21. Kanda N and Watanabe S (2001) Gangliosides GD1b, GT1b, and GQ1b enhance IL-2 and IFN-gamma production and suppress IL-4 and IL-5 production in phytohemagglutininstimulated human T cells. J Immunol 166, 72-80

22. Hsieh AC, Truitt ML and Ruggero D (2011) Oncogenic AKTivation of translation as a therapeutic target. $\mathrm{Br}$ J Cancer 105, 329-336

23. Juric D, Krop I, Ramanathan RK et al (2017) Phase I dose-escalation study of taselisib, an oral pi3k inhibitor, in patients with advanced solid tumors. Cancer Discov 7, 704-715

24. Dreyling M, Santoro A, Mollica L et al (2017) Phosphatidylinositol 3-Kinase Inhibition by copanlisib in relapsed or refractory indolent lymphoma. J Clin Oncol 35, 3898-3905

25. Raphael J, Desautels D, Pritchard KI, Petkova E and Shah PS (2018) Phosphoinositide 3-kinase inhibitors in advanced breast cancer: A systematic review and meta-analysis. Eur J Cancer 91, 38-46

26. Xu J and Liao K (2004) Protein kinase B/AKT 1 plays a pivotal role in insulin-like growth factor-1 receptor signaling induced 3T3-L1 adipocyte differentiation. J Biol Chem 279,
35914-35922

27. Zhang L, Grennan-Jones F, Lane C, Rees DA, Dayan CM and Ludgate M (2012) Adipose tissue depot-specific differences in the regulation of hyaluronan production of relevance to Graves' orbitopathy. J Clin Endocrinol Metab 97, 653662

28. Kumar S, Nadeem S, Stan MN, Coenen M and Bahn RS (2011) A stimulatory TSH receptor antibody enhances adipogenesis via phosphoinositide 3-kinase activation in orbital preadipocytes from patients with Graves' ophthalmopathy. J Mol Endocrinol 46, 155-163

29. Li B and Smith TJ (2014) PI3K/AKT pathway mediates induction of IL-1RA by TSH in fibrocytes: modulation by PTEN. J Clin Endocrinol Metab 99, 3363-3372

30. Thaiss CA, Levy M, Itav S and Elinav E (2016) Integration of Innate Immune Signaling. Trends Immunol 37, 84-101

31. Kawasaki T and Kawai T (2014) Toll-like receptor signaling pathways. Front Immunol 5, 461

32. Lim HS, Back KO, Kim HJ, Choi YH, Park YM and Kook KH (2014) Hyaluronic acid induces COX-2 expression via CD44 in orbital fibroblasts from patients with thyroid-associated ophthalmopathy. Invest Ophthalmol Vis Sci 55, 7441-7450

33. Kaback LA and Smith TJ (1999) Expression of hyaluronan synthase messenger ribonucleic acids and their induction by interleukin-1 1 beta in human orbital fibroblasts: potential insight into the molecular pathogenesis of thyroid-associated ophthalmopathy. J Clin Endocrinol Metab 84, 4079-4084

34. Zhang L, Bowen T, Grennan-Jones F et al (2009) Thyrotropin receptor activation increases hyaluronan production in preadipocyte fibroblasts: contributory role in hyaluronan accumulation in thyroid dysfunction. J Biol Chem 284, 26447-26455

35. Pasonen-Seppanen S, Takabe P, Edward M et al (2012) Melanoma cell-derived factors stimulate hyaluronan synthesis in dermal fibroblasts by upregulating HAS2 through PDGFRPI3K-AKT and p38 signaling. Histochem Cell Biol 138, 895911

36. Qin J, Berdyshev E, Poirer C, Schwartz NB and Dawson G (2012) Neutral sphingomyelinase 2 deficiency increases hyaluronan synthesis by up-regulation of hyaluronan synthase 2 through decreased ceramide production and activation of Akt. J Biol Chem 287, 13620-13632

37. Kultti A, Karna R, Rilla K et al (2010) Methyl-beta-cyclodextrin suppresses hyaluronan synthesis by down-regulation of hyaluronan synthase 2 through inhibition of Akt. J Biol Chem 285, 22901-22910

38. Manning BD and Cantley LC (2007) AKT/PKB signaling: navigating downstream. Cell 129, 1261-1274 